\title{
Beta-cell replacement
}

\author{
N.M. D esai ${ }^{1}$, G.S. Korbutt ${ }^{2}$, C.F. B arker ${ }^{1}$, K.J. L afferty ${ }^{3}$ \\ ${ }^{1}$ Department of Surgery, University of Pennsylvania School of Medicine, Philadelphia, Pennsylvania, USA \\ ${ }^{2}$ Surgical-Medical Research Institute, University of Alberta, Edmonton, Alberta, Canada \\ ${ }^{3}$ John Curtin School of Medical Research, Canberra, Australia
}

Transplantation of isolated pancreatic islets has been heralded as the ideal therapy for the treatment of patients with insulin-dependent diabetes mellitus (IDDM) since it is a relatively safe procedure which should obviate the need for exogenous insulin therapy. More importantly, the resultant euglycaemia should prevent, and possibly even reverse, the devastating secondary complications of the disease. The Diabetes Control and Complications Trial demonstrated that the optimal control of blood glucose possible with intensive insulin treatment was associated with a reduced risk of developing diabetes related complications (nephropathy, retinopathy, accelerated coronary artery disease, and neuropathy) [1]. However, this aggressive insulin therapy substantially increased the frequency of dangerous hypoglycaemic episodes. Maintenance of tight glucose control over an extended period also required extraordinary patient effort. In contrast, islet transplantation can potentially provide better glucose control than even the best insulin therapy and should do so without the risk of severe hypoglycaemic events.

Over 25 years have passed since the initial demonstration that islet transplantation could completely reverse diabetes in rodents [2]. These early reports provoked considerable interest in patients with the disease and their physicians. Since that time, the once formidable technical and immunologic problems have been solved and long term success of islet allotransplantation in rodents can be achieved using

Participants: R.G. Bretzel, University of Giessen, Germany; C. G. Groth, Karolinska Institute, Sweden; P. J. Morris, University of Oxford, UK; A. Naji, University of Pennsylva;ia, USA; C. Ricordi, University of Miami, Florida, USA

Corresponding author: N. M. Desai, M. D., Department of Surgery, 4 Silverstein, 3400 Spruce Street, Philadelphia, PA 19104, USA standard immunosuppression or by treating islets prior to transplantation. Unfortunately, the results in humans have been poor - almost all islet transplants in IDDM patients have failed. For islet transplantation to become a predictable, successful, and widespread treatment for IDDM, solutions must be found to overcome the need for continuous immunosuppression and for increasing the availability of insulin-producing tissue. The goal of this work group was to: 1) outline the major recent advances in transplantation and promising future directions in the field; 2) review the current status of clinical islet transplantation; 3) discuss strategies for preventing alloimmune and autoimmune destruction of transplanted islets; and 4) evaluate the scientific basis of using xenogeneic pancreatic islets and review the recent regulatory issues that surround the use of xenografts.

Peter Morris: Transplantation: past, present and future

Progress in the field of organ transplantation: Peter Morris reported on the remarkable advancements that have been achieved in the field of transplantation, largely due to improved immunosuppressive therapy. Initial attempts at immunosuppression involved the use of total body irradiation, which had a high mortality rate and a narrow therapeutic window. In 1963 the combined use of azathioprine and prednisone began, which allowed a 30-40\% survival of cadaveric renal allografts. In the early 1980 s the immunosuppressive agent cyclosporine was introduced. The improvement in results soon made it the agent of choice for all solid organ transplantation. When cyclosporine was used in combination with azathioprine and prednisone for renal transplantation, 5 year graft survival became nearly $80 \%$. An equally dramatic 
effect with cyclosporine was observed in liver transplantation by Tom Starzl at the University of Pittsburgh. More recently, the use of tacrolimus (FK506) in place of cyclosporine in liver transplantation has been shown to decrease the incidence of rejection episodes and perhaps improve long term graft survival.

Whole pancreas transplantation was first performed at the University of Minnesota in 1966 in an IDDM patient. This major operative procedure involves a substantial risk of morbidity and mortality, with nearly $30 \%$ of patients suffering a complication that requires prolonged hospitalization. However successful pancreas transplants have been shown in some cases to stabilize diabetic retinopathy and reverse diabetic neuropathy. An overall success rate of about $70 \%$ is being reported for whole pancreas transplantation while some groups such as Hans Solinger's at the University of Wisconsin have achieved an $80 \%$ pancreas graft survival at 5 years [3].

The penalties of immunosuppression: Despite the impressive improvements in transplantation over its 45 year history there is still much to be accomplished before this form of therapy can be justified in other than life saving circumstances. The penalty of increasingly potent immunosuppressive agents is evidenced by the high prevalence of cardiovascular disease and malignancy in long-surviving patients. In the United Kingdom, a study of transplant patients on chronic immunosuppression demonstrated a dramatic increase in mortality secondary to cardiovascular disease when compared to age-matched control subjects from the general population. The Australian registry has tracked the incidence of cancers developing in patients on long term immunosuppression after an organ transplant. After 20 years of immunosuppressive therapy, over $60 \%$ of patients had developed a cancer of some type, with over $20 \%$ having a non-skin malignancy. These complications clearly demonstrate the long term effects of immunosuppression and highlight some of the limitations of organ transplantation.

These data also argue for caution in the employment of either whole pancreas or islet transplantation for the treatment of diabetic patients. Given the complications of current immunosuppressive agents, it is unacceptable to expose most IDDM patients to these medications unless they are obligated to receiving immunosuppression for a kidney transplant. Only when transplantation can be performed with safer immunosuppression will routine employment of this treatment be justified in diabetic patients prior to the onset of complications. This is particularly unfortunate since the prevention of complications (and not their treatment) is the very purpose of pancreas and islet transplantation.
Reinhard Bretzel, Camillo Ricordi: Clinical islet transplantation

Current status of islet autotransplantation and allotransplantation: The International Islet Transplant Registry was established in 1989 at the University of Giessen, Germany and is directed by Reinhard Bretzel. Dr. Bretzel reviewed the registry data at this meeting [4]. Recipients with pancreatectomy-induced diabetes mellitus (PIDM) receiving an islet autograft exhibited a $67 \%$ incidence of insulin independence at 1 year post-transplantation. These data clearly demonstrate that long term insulin independence can be routinely achieved with currently available techniques for isolating, purifying, and transplanting human islets. The frequency of insulin independence at 1 year post-transplantation in the same PIDM group of patients treated with islet allografts rather than autografts was $40 \%$. However in IDDM recipients engrafted with islet allografts, only a $7 \%$ success rate has been reported to the registry, suggesting that recurrent autoimmunity leads to islet destruction. In these patients, establishment of insulin independence was facilitated if: 1) islets were isolated from pancreata with a mean preservation time less than $8 \mathrm{~h}$;2) more than 6000 islet equivalents per $\mathrm{kg}$ bodyweight were transplanted; 3 ) islets were transplanted into the liver via the portal vein; and 4) induction immunosuppression included antilymphocyte or antithymocyte globulin. In recipients meeting all four criteria, $70 \%$ showed basal C-peptide levels 1 $\mathrm{ng} / \mathrm{ml}$ or more, $83 \%$ had $\mathrm{HbA}_{1 \mathrm{c}}$ levels under $7 \%$, and $20 \%$ were insulin independent at 1 year followup.

I slet transplantation at the U niversity of G iessen: In order to increase the likelihood of achieving insulin independence, the clinical islet transplant programme at the University of Giessen implemented strategies in their transplant protocol which were previously shown in experimental animal models to promote islet engraftment. These included induction therapy with anti-T-cell antibodies 3 days prior to transplantation, maintaining whole blood cyclosporine trough levels between $300-400 \mathrm{ng} / \mathrm{ml}$ for 3 months posttransplant, careful control of hyperglycaemia posttransplantation with intravenous insulin, and peritransplant treatment with nicotinamide, verapamil, and pentoxifylline. A total of $38 \mathrm{C}$-peptide negative IDDM patients were given transplants by the Giessen group with the majority of patients (33/38) receiving intraportal grafts isolated from a single donor pancreas. In 17 of these patients, islets were implanted simultaneously with a kidney (SIK), 16 patients received the islets after a previous kidney allograft (IAK), and in 5 cases islets were transplanted alone. The 1 year follow-up in a total of 8 SIK and 14 IAK transplants was reported. The combined 1 year 
patient and kidney graft survival in the SIK and IAK transplants was 100 and $93 \%$, respectively -1 patient in the IAK group died of a myocardial infarction 14 days after islet transplantation. Moreover, no acute kidney rejection episodes were observed in either group of patients. At 12 months post-transplantation, 7 of $8(88 \%)$ SIK recipients and 7 of 13 $(54 \%)$ IAK patients exhibited evidence of islet allograft function as determined by a C-peptide level 1 $\mathrm{ng} / \mathrm{ml}$ or more, while ongoing insulin independence was achieved in 2 SIK and 3 IAK recipients between 280 and 400 days post-transplantation. The 8 patients receiving SIK transplants exhibited better glucose control when compared to a control group of 8 IDDM patients with kidney transplants alone (KTA) as evidenced by $\mathrm{HbA}_{1 \mathrm{c}}$ levels (6.4 vs $7.9 \%$, respectively). Furthermore none of the SIK recipients experienced severe hypoglycaemic episodes after islet transplantation (vs 2.6 episodes pre-transplant), whereas the KTA group averaged more than 2 severe hypoglycaemic episodes per year both pre- and posttransplantation.

Intraportal islet transplantation alone was also performed in IDDM recipients prone to severe hypoglycaemia in whom defective glucose counterregulation and hypoglycaemia unawareness had been documented. Prior to islet transplantation, glucagon and epinephrine responses to hypoglycaemia were absent or severely impaired in all patients undergoing stepwise hypoglycaemic clamp testing. Autonomic warning symptoms were also absent in all patients during hypoglycaemia. Following transplantation, patients were given a limited course of immunosuppression which consisted of cyclosporine and anti-CD4 antibody for 30 days. One month after transplantation, these patients were retested and found to have no improvement in the glucagon response. However, epinephrine, norepinephrine and cortisol responses were improved in all patients. Moreover, all patients now had autonomic warning symptoms. These results demonstrate that islet transplantation does not restore hypoglycaemia-induced glucagon secretion, however it does improve the response of most counterregulatory hormones and hypoglycaemia awareness even in patients with long-standing IDDM.

These data from the University of Giessen are clearly the best results that have been achieved thus far with islet transplantation in IDDM recipients. They provide evidence that early human islet allograft failure can be consistently prevented by refining peri-transplant management and that insulin independence can be achieved in a significant portion of the patients with the islet mass isolated from a single donor pancreas. These results represent a major step forward; however further research is needed in the development of immunosuppressive protocols optimized for islet engraftment. It should also be mentioned that these results have been achieved in
IDDM patients who are obligated to chronic immunosuppression for a previous or simultaneous kidney transplant, and that this therapy is not currently appropriate for IDDM patients in the early stages of the disease.

The future of clinical isl et transplantation: Dr. Ricordi described how protocols must now be developed in clinical islet transplantation to avoid the need for continuous recipient immunosuppression, which severely limits the potential application of this approach at earlier stages of IDDM. It has been commonly believed that if islet transplantation is performed early on, diabetes related complications are prevented or reduced. Camillo Ricordi indicated that the lesson learned in the 1980 s was that in order to achieve euglycaemia after an islet allograft, a greater islet cell mass needed to be transplanted. Dr. Ricordi's automated method of islet isolation allowed workers to reproducibly purify human islets in large numbers, and with an adequate beta-cell mass, a somewhat increased frequency of patients achieved normoglycaemia after transplantation. However, the number of patients with insulin independence has not dramatically improved in the $1990 \mathrm{~s}$, and the message for this decade is to avoid primary non-function immediately after islet transplantation and eliminating the need for chronic immunosuppression.

Dr. Ricordi suggested that in those patients undergoing upper abdominal exenteration for advanced malignancies and simultaneous liver-islet transplantation, the relatively high rate of insulin independence was related to the fact that the islets were placed in an autologous environment. In contrast when islets are placed in an non-autologous liver, the damage they have suffered from isolation and ischaemia probably increases their immunogenicity, thereby causing more vigorous rejection. He suggested that treatment of islet allograft recipients with nicotinamide and verapamil may reduce ischaemic injury and the resultant primary non-function in the immediate post-transplant period.

Infusion of donor bone marrow cells (DBMC) is a strategy which is currently being tested in clinical trials to enhance the survival of human islet allografts. These trials were prompted by recent encouraging results obtained in liver transplant recipients who were treated with DBMC infusions [5]. For example, when liver transplantation was combined with DBMC infusions on days 5 and 11, graft survival was $89 \%$ at 20 months follow-up compared to only $72 \%$ in the control group. However, one disadvantage of this approach is the higher incidence of graft versus host disease in these patients. Dr. Ricordi also reported that insulin independence has been achieved in two patients receiving kidney-islet grafts in combination with DBMC infusion, although these patients had impaired glucose tolerance during oral 
Table 1. Strategies to promote islet allograft survival in the absence of immunosuppression

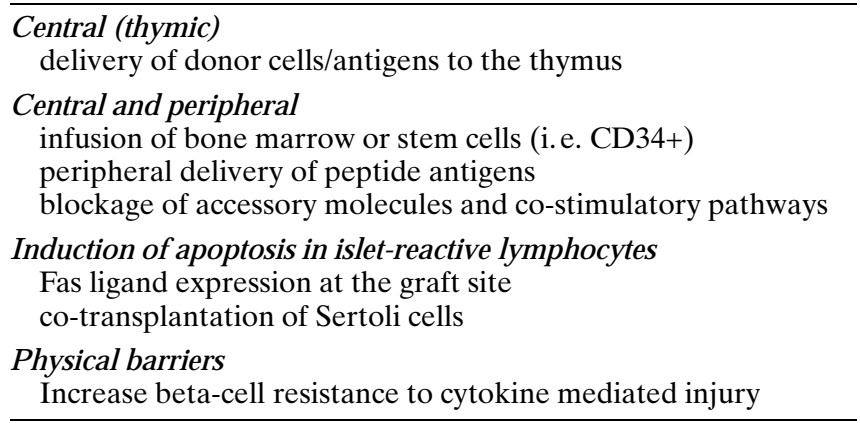

glucose challenge. These preliminary observations suggest the possibility for improved allograft survival with DBMC infusion, but the potential for avoiding chronic immunosuppression remains to be demonstrated.

A partial list of methods currently under investigation for the prevention of islet allograft rejection is given in Table 1. One recent approach is the co-transplantation of allogeneic islets with Fas ligand secreting cells, as published in two separate models by Lau and by Korbutt $[6,7]$. This method was of great interest to members of the work group who felt that it was promising and deserved further study.

Peter Morris, Ali Naji: Strategies to prevent immune destruction of islet transplants

Tolerance: Dr. Morris discussed how the complications associated with global immunosuppression of the recipient would be solved by the achievement of specific tolerance to alloantigens. Tolerance is the "holy grail" of transplantation biologists. It can currently be achieved in rodent models via a variety of protocols. It is hoped that clinical trials will soon allow for tolerance to be successfully produced in IDDM patients receiving an islet transplant.

The protocol being used in the laboratory by Katherine Wood at the University of Oxford involves the pretreatment of rodents with anti-CD4 antibody along with donor specific transfusion, followed by the placement of a heart allograft 4 weeks later. These animals not only accept the heart allografts, but also become tolerant, as evidenced by the acceptance of skin grafts. This tolerance state is dependent upon the presence of the allograft itself for it does not persist if the heart graft is removed. This group plans to apply this model using a non-depleting form of anti-CD4 antibody in a clinical model of renal transplantation in the near future.

A li Naji: Intrathymic tolerance: The realization that the thymus serves as the primary site for the initiation of self-tolerance led to experiments several years ago to evaluate the impact of implanting foreign islets in the thymus as a method of promoting tolerance to allografts [8]. In initial experiments, allogeneic islets were inoculated into the thymus of adult rat recipients that were briefly immunosuppressed with a single dose of antilymphocytic serum. Not only did these islet allografts survive indefinitely, but they also induced a state of donor specific unresponsiveness that allowed for the survival of a second islet allograft under the kidney capsule without immunosuppression. Subsequently it was found that lymphoid donor cells injected directly into the thymus would also promote donor specific tolerance to allografts of islets or other tissues. Brayman, at the University of Pennsylvania, has applied intrathymic islet transplantation to large animals using pancreatectomized dogs that received a temporary course of cyclosporine. Animals with intrathymic islets maintained normoglycaemia for a mean of 102 days compared to 65 days for animals with intraportal islets. These initial results appear promising and deserve further investigation.

L ocal immunosuppression: Instead of the systemic administration of immunosuppressive agents and its associated complications, the local delivery of immunosuppression at the site of an islet graft might minimize the dose needed and the resultant side effects. One way to employ this strategy would be to induce transplanted islets to produce their own immunosuppressive cytokines, such as interleukin (IL)-10 and transforming growth factor (TGF) $\beta$, or to have these agents produced at the transplantation site by other cells. Adenoviral vectors were used as a gene delivery system to test this hypothesis. When a combination of IL-10 and TGF- $\beta$ was employed to transfect mouse islets, nearly $40 \%$ of allografts survived beyond 100 days. These results not only demonstrate that islets can be transfected with adenoviral vectors without significantly damaging them, but also that local immunosuppression can prolong allograft survival. Thus the use of gene therapy to modify islets prior to transplantation appears to be promising, but this method will require further study to find the optimal gene product for overcoming rejection.

D estruction of islet allografts by disease recurrence: Transplanted islet allografts in IDDM recipients are exquisitely vulnerable to immune mediated destruction. In rodent models, the susceptibility of transplanted islets to allograft rejection is second perhaps only to that of skin allografts. However, several methods of overcoming the allograft response are quite satisfactory, at least in rodents. Recurrence of autoimmune disease; i.e. the destruction of the islet transplant by the original diabetogenic immune response, is a substantial additional immune barrier as initially observed in the BioBreeding (BB) rat model. 
Subsequently, recurrence has also been shown in the non-obese diabetic (NOD) mouse and in IDDM patients [9]. Even when islets are cultured in vitro prior to transplantation, a manoeuver that eliminates alloreactivity, they are rapidly destroyed in autoimmune rodents. These results indicate that disease recurrence is a potent mediator of islet allograft destruction in experimental models. Recurrence may also be the major reason for the poor results of islet transplantation that have been observed in IDDM patients.

Recent studies in Dr. Naji's laboratory provide new insights into the pathogenesis of autoimmunity in the NOD mouse model of diabetes. While it has long been accepted that both $\mathrm{CD}^{+}{ }^{+}$and $\mathrm{CD} 8{ }^{+} \mathrm{T}$ cells are implicated in the pathogenesis of diabetes in the NOD mouse, the role of the B lymphocyte has not been well studied. However, Noorchasm from the University of Pennsylvania presented data that demonstrate $\mathrm{B}$ cells to be required for the initiation of autoimmune diabetes. This study involved the use of anti- $\mu$ chain antibody to induce in vivo depletion of B lymphocytes in NOD mice starting at birth. They found that B cell depletion completely abrogates the development of insulitis and diabetes in these mice.

These studies demonstrate the vast complexity of the autoimmune process in IDDM and that many factors remain to be elucidated regarding the pathogenesis of this disease. Moreover, it is clear that recurrence of disease will be a major barrier to success in clinical islet transplantation. Further research into the autoimmune process and means to overcome recurrence after islet transplantation is sorely needed.

\section{Carl Groth: Xenotransplantation}

Porcine islet xenotransplantation: If islet transplantation is to become a widespread treatment for IDDM, it is clear that the supply of human donor organs will become a major limitation. In an attempt to overcome this organ shortage, islets from animal sources are being considered for xenotransplantation. Pigs are potentially an excellent source for xenogeneic islets since they are readily available and breed easily in captivity, producing large litters. Porcine insulin is structurally similar to human insulin and has been used for the treatment of IDDM patients for years. Ethical concerns over the use of swine are minimal since they are raised for food consumption.

Dr. Groth outlined a clinical study conducted at the Karolinska Institute, Sweden in which 8 IDDM patients who were recipients of a prior kidney allograft were transplanted intraportally with xenogeneic fetal porcine islet cell clusters [10]. All recipients received induction immunosuppression with either anti-thymocyte globulin or 15-deoxyspergualin, followed by standard triple-drug immunosuppression. In 4 patients, small amounts of porcine C-peptide were detectable in the urine for as long as 400 days after transplantation. In addition, two uraemic IDDM patients were transplanted with fetal porcine islet cell clusters under the capsule of a simultaneously transplanted renal allograft. Immunosuppression in these individuals was the same as the first group of patients. At 3 weeks post-transplantation, a graft biopsy revealed the presence of insulin and glucagon positive cells containing only a sparse infiltrate of eosinophils and mononuclear cells. However, insulin requirements in these patients were not reduced. Interestingly, all the patients developed high levels of xenoreactive antibodies recognizing galactosyl $\alpha 1-3$ galactosyl ( $\alpha$-Gal) antigens. These antigens are normally expressed by the endothelium and render a vascularized xenograft susceptible to complement mediated hyperacute rejection. A major topic of discussion by the work group was whether a non-vascularized porcine islet xenograft is susceptible to hyperacute rejection. Some members felt that since islets are not immediately vascularized, hyperacute rejection would not be a problem. However other members stated that since porcine islet endocrine cells also express $\alpha$-Gal, they may also be susceptible to hyperacute rejection. This opinion is supported by the finding by one group that porcine islets are destroyed within $12 \mathrm{~h}$ when transplanted into primate hosts. Clearly further investigation is needed into the susceptibility of islet xenografts to hyperacute rejection.

R egulation of xenotransplantation: Dr. Groth also reviewed the recent developments in the regulatory process of xenotransplantation. In the United Kingdom, the government has recently placed a moratorium on the use of xenogeneic tissues in man. This restriction is largely based upon the findings that a porcine endogenous retrovirus (PERV) could infect human cells in vitro. There is a concern that following xenotransplantation a PERV could replicate in a recipient and potentially spread to close contacts, putting all at risk for retroviral induced cancers [11]. A recent report in the United Kingdom on xenotransplantation by the Nuffield Council on Bioethics favours banning xenotransplantation due to the risk of zoonoses such as PERVs. This report also states that our current knowledge of xenograft rejection is too limited to justify clinical trials, and that we do not know whether the physiologic function of a xenogeneic organ will substitute for a human organ. Therefore it was concluded that further research was critical before clinical trials of xenotransplantation should be embarked upon.

In the United States there are presently no governmental restrictions that limit xenotransplantation, although the Public Health Service has established a 
set of guidelines. The United States government has allowed the approval process for clinical trials of xenotransplantation to remain with institutional review boards. In fact, five clinical programmes that use xenogeneic sources of tissue have presently been approved in the United States including transplantation of encapsulated porcine islets in IDDM patients. Nonetheless, before an animal source of islets can be considered for transplantation into humans, many concerns need to be addressed.

\section{Future directions and recommendations}

The recent clinical experience with islet transplantation is encouraging; however success rates still lag behind those of solid organ transplantation. Currently islet transplantation is only appropriate for IDDM patients who are also recipients of a kidney transplant, and thus are obligated to chronic immunosuppression. The pitfalls of islet transplantation combined with the high penalty of chronic immunosuppression make this therapy inappropriate for IDDM patients in the early stages of the disease. In order for islet transplantation to become a more widespread treatment, several concerns must be addressed:

- Immunosuppressive protocols must be developed to optimize patient safety and promote islet allograft survival.

- Further basic research is required into the transplantation of islets without systemic immunosuppression, such as tolerance induction, the use of gene therapy to provide a local immunosuppressive environment, and the use of Fas ligand expression at the transplant site to induce apoptosis in islet reactive lymphocytes.

- A better understanding of the autoimmune process in IDDM is vital in order to overcome the obstacle of disease recurrence following islet transplantation.

- Ultimately, an animal source of insulin-producing tissue (such as swine) will be required for the widespread application of islet transplantation. However whether cellular xenogeneic islet grafts will be susceptible to hyperacute rejection is one issue that must be thoroughly examined.

- The safety and regulatory issues encompassing the use of animal tissue for clinical transplantation is a growing area of concern that will certainly impede future clinical trials of islet xenotransplantation.

\section{References}

1. Diabetes Control and Complications Trial Research Group (1993) The effect of intensive treatment of diabetes on the development and progression of long-term complications in insulin-dependent diabetes mellitus. N Engl J Med 329: 977-986

2. Reckard CR, Ziegler MM, Barker CF (1973) Physiological and immunological consequences of transplanting isolated pancreatic islets. Surgery 74: 91-99

3. Sollinger HW, Ploeg RJ, Eckhoff DE et al. (1993) Two hundred consecutive simultaneous pancreas-kidney transplants with bladder drainage. Surgery 114: 736-743

4. Hering BJ, Brendel MD, Schultz AO, Schultz B, Bretzel RG (1996) International islet transplant registry. Newsletter 6: $1-20$

5. Ricordi C, Karatzas T, Nery J et al. (1997) High-dose donor bone marrow infusions to enhance allograft survival: the effect of timing. Transplantation 63: 7-11

6. Lau HT, Yu M, Fontana A, Stoeckert CJ (1996) Prevention of islet allograft rejection with engineered myoblasts expressing FasL in mice. Science 273: 109-112

7. Korbutt GS, Elliot JF, Rajotte RV (1997) Cotransplantation of allogeneic islets with allogeneic testicular cell aggregates allows long-term graft survival without systemic immunosuppression. Diabetes 46: 317-322

8. Posselt AM, Barker CF, Tomaszewski JE, Markmann JF, Choti MA, Naji A (1990) Induction of donor-specific unresponsiveness by intrathymic islet transplantation. Science 249: 1293-1295

9. Stegall MD, Lafferty KJ, Kam I, Gill RG (1996) Evidence of recurrent autoimmunity in human allogeneic islet transplantation. Transplantation 61: 1272-1274

10. Groth CG, Korsgren O, Tibell A et al. (1994) Transplantation of porcine fetal pancreas to diabetic patients. Lancet 344: 1402-1404

11. Patience C, Takeuchi Y, Weiss RA (1997) Infection of human cells by an endogenous retrovirus of pigs. Nature Medicine 3: 282-286 